\title{
Transfer of Bacillus alginolyticus, Bacillus chondroitinus, Bacillus curdlanolyticus, Bacillus glucanolyticus, Bacillus kobensis, and Bacillus thiaminolyticus to the Genus Paenibacillus and Emended Description of the Genus Paenibacillus
}

\author{
OSAMU SHIDA,${ }^{1 *}$ HIROAKI TAKAGI,${ }^{1}$ KIYOSHI KADOWAKI,${ }^{1}$ LAWRENCE K. NAKAMURA, ${ }^{2}$ \\ AND KAZUO KOMAGATA ${ }^{3}$
}

Research Laboratory, Higeta Shoyu Co., Ltd., Choshi, Chiba $288,{ }^{1}$ and Department of Agricultural Chemistry, Faculty of Agriculture, Tokyo University of Agriculture, Setagaya-ku, Tokyo $156,{ }^{3}$ Japan, and Microbial Properties Research, National Center for Agricultural Utilization Research, U.S. Department of Agriculture, Peoria, Illinois $61604^{2}$

\begin{abstract}
We determined the taxonomic status of six Bacillus species (Bacillus alginolyticus, Bacillus chondroitinus, Bacillus curdlanolyticus, Bacillus glucanolyticus, Bacillus kobensis, and Bacillus thiaminolyticus) by using the results of 16S rRNA gene sequence and cellular fatty acid composition analyses. Phylogenetic analysis clustered these species closely with the Paenibacillus species. Like the Paenibacillus species, the six Bacillus species contained anteiso- $\mathrm{C}_{15: 0}$ fatty acid as a major cellular fatty acid. The use of a specific PCR primer designed for differentiating the genus Paenibacillus from other members of the Bacillaceae showed that the six Bacillus species had the same amplified 16S rRNA gene fragment as members of the genus Paenibacillus. Based on these observations and other taxonomic characteristics, the six Bacillus species were transferred to the genus Paenibacillus. In addition, we propose emendation of the genus Paenibacillus.
\end{abstract}

Rod-shaped, aerobic, endospore-forming bacteria have generally been assigned to the genus Bacillus, a systematically diverse taxon (5). 16S rRNA gene sequence analyses have identified at least 10 phylogenetic groups in the genus Bacillus $(2,3,7,23,26,31,33,34,36)$. Five of the groups have been reclassified as the new genera Alicyclobacillus (36), Paenibacillus (2), Halobacillus (33), Brevibacillus (31), and Aneurinibacillus (31). The genus Paenibacillus as proposed by Ash et al. (2) consists of the following 11 species: Paenibacillus polymyxa, Paenibacillus alvei, Paenibacillus gordonae, Paenibacillus larvae, Paenibacillus pulvifaciens, Paenibacillus macerans, Paenibacillus azotofixans, Paenibacillus pabuli, Paenibacillus macquariensis, Paenibacillus amylolyticus, and Paenibacillus validus. Clostridium durum has also been transferred to this genus (6). Recently, Heyndrickx et al. reported that $P$. gordonae was a synonym of $P$. validus (12), that $P$. pulvifaciens was a subspecies of P. larvae (11), and that Bacillus lautus and Bacillus peoriae should be transferred to the genus Paenibacillus (13). In addition, Nakamura proposed the new species Paenibacillus apiarius (22). Consequently, the genus Paenibacillus consists of 13 species and one subspecies.

Members of the genus Paenibacillus are facultatively anaerobic organisms that produce spores in definitely swollen sporangia and have $\mathrm{G}+\mathrm{C}$ contents ranging from 45 to $54 \mathrm{~mol} \%$, and some of these organisms excrete diverse assortments of extracellular polysaccharide-hydrolyzing enzymes $(5,25)$. Interestingly, many recently described Bacillus species possess the general characteristics of the genus Paenibacillus. The noteworthy ability of these species to hydrolyze complex carbohydrates, including alginate (19), chondroitin (19), chitin (25), curdlan (14), and other polysaccharides (25), suggests that some of them may be related to the genus Paenibacillus. To understand the taxonomic position of these Bacillus species

\footnotetext{
* Corresponding author. Mailing address: Research Laboratory, Higeta Shoyu Co., Ltd., 2-8 Chuo-cho, Choshi, Chiba 288, Japan. Phone: 81-479-22-1180. Fax: 81-479-24-3422. E-mail: LDX05744 @niftyserve.or.jp.
}

among the Bacillaceae, we determined the sequences of their 16S rRNA genes and compared these sequences with homologous sequences available for other members of the Bacillaceae. In addition, a highly specific PCR amplification primer was designed on the basis of the $16 \mathrm{~S}$ rRNA gene sequence alignments for differentiating the genus Paenibacillus from other aerobic, endospore-forming rods.

\section{MATERIALS AND METHODS}

Bacterial strains. The bacterial strains used in this study are listed in Table 1. Working stocks were cultured on tryptic soy agar plates (Difco Laboratories, Detroit, Mich.) for $24 \mathrm{~h}$ at $37^{\circ} \mathrm{C}$. The strains were stored at room temperature. Sequencing the 16S rRNA genes. The methods used for preparation of chromosomal DNA and PCR amplification of the 16S rRNA gene from chromosoma DNA and the primers used for PCR amplifications have been described previously (31). Amplified 16S rRNA genes purified with a QIAquick Spin PCR purification kit (QIAGEN GmbH, Hilden, Germany) were used for sequencing templates. Sequencing was carried out as described by Sanger et al. (29) by using a Dye terminator cycle sequencing FS Ready Reaction kit (Perkin-Elmer Co., Foster City, Calif.) and a model ABI 373A automatic DNA sequencer (PerkinElmer $\mathrm{Co}$.). The seven sequencing primers used have been described by Fox et al. (9).

Comparison of 16S rRNA gene sequences. Sequences determined in this study were compared with $16 \mathrm{~S}$ rRNA gene sequences obtained from the EMBL GenBank, and DDBJ databases. Multiple alignment of sequences, calculation of nucleotide substitution rates ( $K_{\text {nuc }}$ values) (15), construction of a neighborjoining phylogenetic tree (28), and a bootstrap analysis with 1,000 replicates for evaluation of phylogenetic tree topology (8) were carried out with the CLUSTAL $\mathrm{W}$ version 1.5 program (35). Alignment gaps and unidentified base positions were not taken into account for the calculations.

Cellular fatty acid compositions. Cells of all of the strains listed in Table 1 except the $P$. macquariensis strains were cultivated overnight in tryptic soy broth (Difco Laboratories) at $37^{\circ} \mathrm{C}$. The $P$. macquariensis strains were cultured in tryptic soy broth overnight at $23^{\circ} \mathrm{C}$. Preparation and determination of cellular fatty acids were carried out as described by Komagata and Suzuki (16).

Identification of Paenibacillus strains by 16S rRNA gene amplification. Strains belonging to the genus Paenibacillus were identified by 16 S rRNA gene PCR amplification by using specific forward primer PAEN515F (5'-GCTCGGAGA GTGACGGTACCTGAGA-3') and universal reverse primer 1377R. The sequences of universal forward primer $27 \mathrm{FC}$ and reverse primer $1377 \mathrm{R}$ and the methods used for PCR amplification of the 16S rRNA gene from chromosomal DNA with a detection primer and detection of PCR products have been described previously (31). 


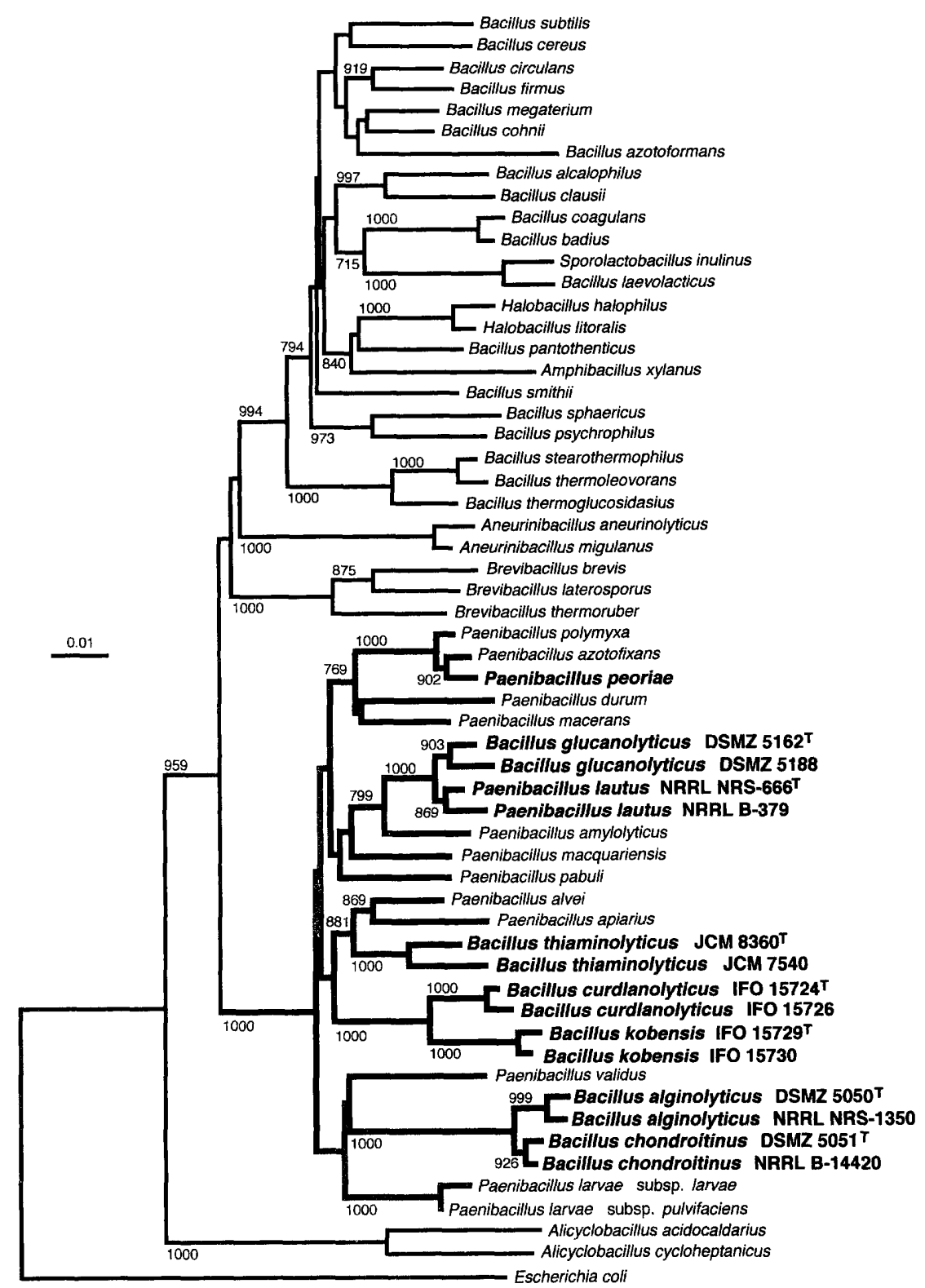

FIG. 1. Phylogenetic relationships of Paenibacillus species and some aerobic, rod-shaped, endospore-forming bacteria based on $16 \mathrm{~S}$ rRNA gene sequences. The branching pattern was generated by the neighbor-joining method. The numbers indicate bootstrap values greater than 700 . Boldface lines and boldface type indicate the cluster consisting of Paenibacillus species and the species sequenced in this study, respectively. Bar $=0.01$ nucleotide substitution per site.

Nucleotide sequence accession numbers. The 16S rRNA gene sequences determined in this study have been deposited in the EMBL, GenBank, and DDBJ databases under the accession numbers listed in Table 1.

\section{RESULTS}

Phylogenetic relationship. We determined the nucleotide sequences (1,393 to 1,437 bases) of the $16 \mathrm{~S}$ rRNA gene from two strains (the type strain and a sensu stricto strain) of $B a$ cillus alginolyticus, Bacillus chondroitinus, Bacillus curdlanolyticus, Bacillus glucanolyticus, Bacillus kobensis, Bacillus thiaminolyticus, and Paenibacillus lautus and the type strain of Paenibacillus peoriae. The intraspecies similarity values for the six Bacillus species and P. lautus were greater than $97.1 \%$. 16S

FIG. 2. Sequence of the detection primer for members of the Paenibacillus cluster (PAEN515F) and alignment of the 16S rRNA gene sequences of Paenibacillus species and some related taxa. Dashes indicate nucleotides identical to those of primer PAEN515F.
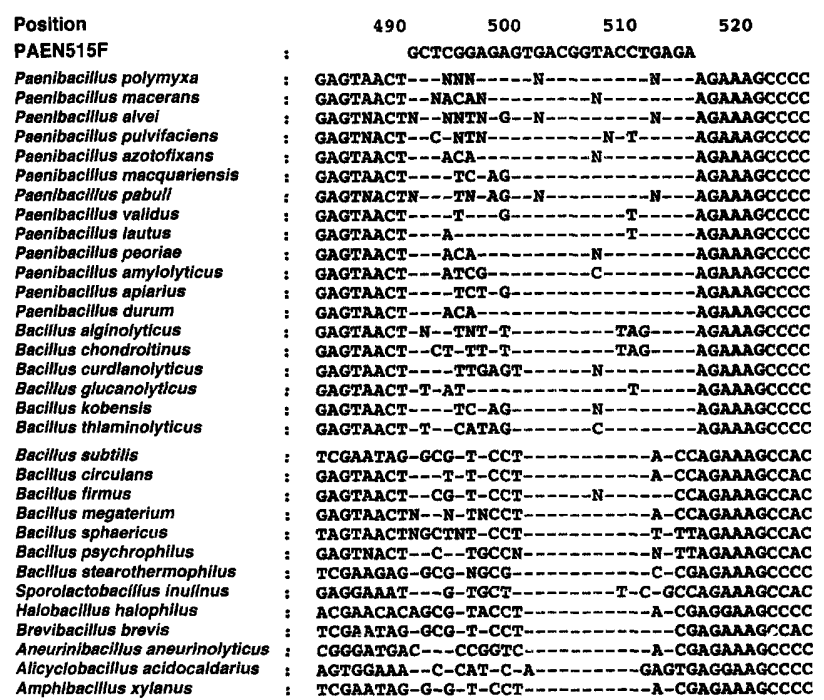
TABLE 1. Bacterial strains used in this study

\begin{tabular}{|c|c|c|c|}
\hline $\operatorname{Strain}^{a}$ & Source $^{b, c}$ & History ${ }^{c}$ & $\begin{array}{l}\text { Accessior } \\
\text { no. }\end{array}$ \\
\hline \multicolumn{4}{|l|}{ Bacillus alginolyticus strains } \\
\hline DSMZ $5050^{\mathrm{T}}$ & 1 & NRRL NRS- $1347^{\mathrm{T}}$ from N. R. Smith from F. E. Clark strain $3^{\mathrm{T}}\left(=\right.$ HSCC $175^{\mathrm{T}}$ ) & D78465 \\
\hline NRRL NRS-1350 & 2 & N. R. Smith from F. E. Clark strain 8 (= HSCC 609) & D88517 \\
\hline \multicolumn{4}{|l|}{ Bacillus chondroitinus strains } \\
\hline DSMZ 5051 & 1 & NRRL NRS- $1351^{\mathrm{T}}$ from N. R. Smith from F. E. Clark strain $12^{\mathrm{T}}\left(=\right.$ HSCC $\left.176^{\mathrm{T}}\right)$ & D82064 \\
\hline NRRL B-14420 & 2 & L. K. Nakamura, isolated from soil (= HSCC 612 ) & D88518 \\
\hline \multicolumn{4}{|l|}{ Bacillus curdlanolyticus strains } \\
\hline IFO $15724^{\mathrm{T}}$ & 3 & Y. Kanzawa strain YK9 $9^{\mathrm{T}}$, isolated from soil $\left(=\operatorname{HSCC} 491^{\mathrm{T}}\right)$ & D78466 \\
\hline IFO 15726 & 3 & Y. Kanzawa strain YK161, isolated from soil (= HSCC 870) & D88515 \\
\hline \multicolumn{4}{|l|}{ Bacillus glucanolyticus strains } \\
\hline DSMZ $5162^{\mathrm{T}}$ & 1 & F. G. Priest strain $\mathrm{S93^{ \textrm {T } }}$ from J. R. Norris strain $\mathrm{B} 0030^{\mathrm{T}}\left(=\mathrm{HSCC} 171^{\mathrm{T}}\right)$ & D78470 \\
\hline DSMZ 5188 & 1 & F. G. Priest strain E28, isolated from garden soil (= HSCC 872) & D88514 \\
\hline \multicolumn{4}{|l|}{ Bacillus kobensis strains } \\
\hline IFO $15729^{\mathrm{T}}$ & 3 & Y. Kanzawa strain YK205 ${ }^{\mathrm{T}}$, isolated from soil $\left(=\mathrm{HSCC} 488^{\mathrm{T}}\right)$ & D78471 \\
\hline IFO 15730 & 3 & Y. Kanzawa strain YK205, isolated from soil (= HSCC 871) & D88516 \\
\hline \multicolumn{4}{|l|}{ Bacillus thiaminolyticus strains } \\
\hline $\mathrm{JCM} 8360^{\mathrm{T}}$ & 4 & AHU $1393^{\mathrm{T}}\left(=\operatorname{HSCC} 148^{\mathrm{T}}\right)$ & D78475 \\
\hline JCM 7540 & 4 & IAM 1034 from K. Arima (= HSCC 197) & D88513 \\
\hline \multicolumn{4}{|l|}{ Paenibacillus lautus strains } \\
\hline NRRL NRS-666 ${ }^{\mathrm{T}}$ & 2 & University of Washington, "Bacillus lautus" (= HSCC $493^{\mathrm{T}}$ ) & D78473 \\
\hline NRRL B-379 & 2 & N. R. Smith, migratory colonies 1 , isolated from soil (= NRS-676 = HSCC 424) & D85394 \\
\hline \multicolumn{4}{|l|}{ Paenibacillus peoriae strains } \\
\hline IFO $15541^{\mathrm{T}}$ & 3 & $\begin{array}{l}\text { NRRL B-14750 from NRRL BD-5 } 57^{\mathrm{T}} \text { from B. Delaporte strain } 11 . B .9^{\mathrm{T}} \text {, isolated from } \\
\text { soil }\left(=\text { HSCC } 353^{\mathrm{T}}\right)\end{array}$ & D78476 \\
\hline \multirow{2}{*}{\multicolumn{4}{|c|}{ Paenibacillus alvei strains }} \\
\hline & & & \\
\hline IFO $3343^{\mathrm{T}}$ & 3 & $\begin{array}{l}\text { IMAB B-3-4 } 4^{\mathrm{T}} \text { from ATCC } 6344^{\mathrm{T}} \text { from N. R. Smith strain } 662^{\mathrm{T}} \text { from A. G. Lochhead } \\
\text { strain } 127^{\mathrm{T}}\left(=\mathrm{HSCC} 146^{\mathrm{T}}\right)\end{array}$ & \\
\hline NRRL NRS-811 & 2 & J. R. Porter from A. G. Lochhead (= HSCC 897) & \\
\hline \multicolumn{4}{|l|}{ Paenibacillus amylolyticus strains } \\
\hline NRRL B-377 ${ }^{\mathrm{r}}$ & 2 & N. R. Smith from K. F. Kellerman $\left(=\mathrm{NRS}-290^{\mathrm{T}}=\right.$ HSCC $\left.374^{\mathrm{T}}\right)$ & \\
\hline NRRL B-142 & 2 & FDA strain PCI221 (= HSCC 442) & \\
\hline \multicolumn{4}{|l|}{ Paenibacillus apiarius strains } \\
\hline NRRL NRS-1438 ${ }^{\mathrm{T}}$ & 2 & H. Katznelson strain $B \times 3^{\mathrm{T}}$, isolated from honeybee larvae $\left(=\mathrm{HSCC} 603^{\mathrm{T}}\right)$ & \\
\hline NRRL NRS-1578 & 2 & W. C. Haynes (= HSCC 604) & \\
\hline \multicolumn{4}{|l|}{ Paenibacillus azotofixans strains } \\
\hline NRRL B-14372 ${ }^{\mathrm{T}}$ & 2 & L. Selden strain P3L-S ${ }^{\mathrm{T}}$, isolated from soil $\left(=\right.$ HSCC $\left.379^{\mathrm{T}}\right)$ & \\
\hline NRRL B-14359 & 2 & No information available (= HSCC 898 ) & \\
\hline \multicolumn{4}{|l|}{$\begin{array}{l}\text { Paenibacillus larvae subsp. } \\
\text { pulvifaciens strains }\end{array}$} \\
\hline IFO $15408^{\mathrm{T}}$ & 3 & $\begin{array}{l}\text { NRRL B-3685 from N. R. Smith from J. W. Rouatt from H. Katznelson strain } 670^{\mathrm{T}} \text {, } \\
\text { isolated from dead honeybee (= HSCC } 355^{\mathrm{T}} \text { ) }\end{array}$ & \\
\hline NRRL B-14152 & 2 & G. J. Bonde (= HSCC 443) & \\
\hline \multicolumn{4}{|l|}{ Paenibacillus macerans strains } \\
\hline JCM $2500^{\mathrm{T}}$ & 4 & CCM $2012^{\mathrm{T}}$ from R. E. Gordon $\left(=\operatorname{HSCC} 179^{\mathrm{T}}\right)$ & \\
\hline IAM 1243 & 5 & IFO 3490 from NRRL B-388 (= HSCC 194) & \\
\hline \multicolumn{4}{|l|}{$\begin{array}{l}\text { Paenibacillus macquariensis } \\
\text { strains }\end{array}$} \\
\hline CIP $103269^{\mathrm{T}}$ & 6 & DSMZ $2^{\mathrm{T}}$ from ATCC $23464^{\mathrm{T}}$ from B. J. Marshall, isolated from soil ( $=$ HSCC $358^{\mathrm{T}}$ ) & \\
\hline NRRL NRS-1534 & 2 & T. Gibson from NCTC 10419 from B. J. Marshall (= HSCC 899) & \\
\hline \multicolumn{4}{|l|}{ Paenibacillus pabuli strains } \\
\hline NRRL NRS-924 ${ }^{\mathrm{T}}$ & 2 & J. R. Porter from M. Schieblich, "Bacillus pabuli" (= HSCC $\left.492^{\mathrm{T}}\right)$ & \\
\hline NRRL BD-537 & 2 & L. K. Nakamura, isolated from Canadian soil (= HSCC 422) & \\
\hline \multicolumn{4}{|l|}{ Paenibacillus polymyxa strains } \\
\hline $\mathrm{JCM} 2507^{\mathrm{T}}$ & 4 & CCM $1459^{\mathrm{T}}$ from BUCSAV $162^{\mathrm{T}}\left(=\operatorname{HSCC} 184^{\mathrm{T}}\right)$ & \\
\hline NRRL BD-55 & 2 & B. Delaporte, isolated from soil (= HSCC 416) & \\
\hline Paenibacillus validus strains & & & \\
\hline DSMZ $3037^{\mathrm{T}}$ & 1 & $\begin{array}{l}\text { NRRL NRS- } 1000^{\mathrm{T}} \text { from N. R. Smith from J. R. Porter from G. Bredemann, isolated } \\
\text { from soil (= HSCC } 174^{\mathrm{T}} \text { ) }\end{array}$ & \\
\hline CIP 103498 & 6 & F. Pichinoty strain Q1, isolated from Spanish soil (= HSCC 357) & \\
\hline Bacillus subtilis JCM $1465^{\mathrm{T}}$ & 4 & IAM $12118^{\mathrm{T}}$ from ATCC $6051^{\mathrm{T}}$ from H. J. Cohn strain Marburg $\left(=\right.$ HSCC $\left.182^{\mathrm{T}}\right)$ & \\
\hline Bacillus cereus $\mathrm{JCM} 2152^{\mathrm{T}}$ & 4 & IAM $12605^{\mathrm{T}}$ from NCIB $9373^{\mathrm{T}}$ from R. E. Gordon $\left(=\right.$ HSCC $\left.183^{\mathrm{T}}\right)$ & \\
\hline Bacillus megaterium JCM $2506^{\mathrm{T}}$ & 4 & CCM $2007^{\mathrm{T}}$ from R. E. Gordon $\left(=\right.$ HSCC $\left.181^{\mathrm{T}}\right)$ & \\
\hline Bacillus circulans IAM $12462^{\mathrm{T}}$ & 5 & NCIB $9374^{\mathrm{T}}$ from R. E. Gordon $\left(=\mathrm{HSCC} 161^{\mathrm{T}}\right)$ & \\
\hline Bacillus badius ATCC $14574^{\mathrm{T}}$ & 7 & $\begin{array}{l}\text { R. E. Gordon from N. R. Smith strain } 663^{\mathrm{T}} \text { from B. S. Henry strain } 110^{\mathrm{T}} \text { from M. } \\
\text { Batchelor }\left(=\text { HSCC } 154^{\mathrm{T}}\right)\end{array}$ & \\
\hline Bacillus sphaericus JCM $2502^{\mathrm{T}}$ & 4 & CCM $2120^{\mathrm{T}}$ from R. E. Gordon $\left(=\operatorname{HSCC} 498^{\mathrm{T}}\right)$ & \\
\hline
\end{tabular}


TABLE 1-Continued

\begin{tabular}{|c|c|c|c|}
\hline $\operatorname{Strain}^{a}$ & Source ${ }^{b, c}$ & History ${ }^{c}$ & $\begin{array}{l}\text { Accession } \\
\text { no. }\end{array}$ \\
\hline Bacillus stearothermophilus JCM $2501^{\mathrm{T}}$ & 4 & ATCC $12980^{\mathrm{T}}$ from NCA $26^{\mathrm{T}}\left(=\right.$ HSCC $\left.160^{\mathrm{T}}\right)$ & \\
\hline Bacillus coagulans JCM $2257^{\mathrm{T}}$ & 4 & $\begin{array}{l}\text { IAM } 1115^{\mathrm{T}} \text { from ATCC } 7050^{\mathrm{T}} \text { from N. R. Smith strain } 609^{\mathrm{T}} \text { from J. R. Porter } \\
\text { from B. W. Hammer }\left(=\text { HSCC } 180^{\mathrm{T}}\right)\end{array}$ & \\
\hline Sporolactobacillus inulinus NRIC $1133^{\mathrm{T}}$ & 8 & K. Kitahara $\left(=\operatorname{HSCC} 342^{T}\right)$ & \\
\hline Brevibacillus brevis JCM $2503^{\mathrm{T}}$ & 4 & $\begin{array}{l}\text { DSMZ } 30^{\mathrm{T}} \text { from ATCC } 8246^{\mathrm{T}} \text { from N. R. Smith strain } 604^{\mathrm{T}} \text { from J. R. Porter } \\
\text { from NCTC } 2611^{\mathrm{T}} \text { from W. W. Fors strain } 27 \mathrm{~B}^{\mathrm{T}}\left(=\text { HSCC } 186^{\mathrm{T}}\right)\end{array}$ & \\
\hline $\begin{array}{l}\text { Aneurinibacillus aneurinolyticus } \\
\text { ATCC } 12856^{\mathrm{T}}\end{array}$ & 7 & Y. Ito from R. Kimura $\left(=\operatorname{HSCC} 149^{\mathrm{T}}\right)$ & \\
\hline
\end{tabular}

${ }^{a}$ All strains are sensu stricto strains. $\mathrm{T}=$ type strain.
${ }^{b} 1$, Deutsche Sammlung von Mikroorganismen und Zellkulturen $\mathrm{GmbH}$, Braunschweig, Germany; 2, Agricultural Research Service Culture Collection, National Center for Agricultural Utilization Research, Peoria, Ill.; 3, Institute for Fermentation, Osaka, Japan; 4, Japan Collection of Microorganisms, Saitama, Japan; 5, Institute of Molecular and Cellular Biosciences, The University of Tokyo, Tokyo, Japan; 6, Collection des Bactéries de I'Institut Pasteur, Paris, France; 7, American Type Culture Collection, Rockville, Md.; 8, NODAI Research Institute, Culture Collection Center, Tokyo University of Agriculture, Tokyo, Japan.

${ }^{c}$ HSCC, Culture Collection of the Research Laboratory of Higeta Shoyu Co., Ltd., Chiba, Japan; AHU, Department of Agricultural Chemistry, Hokkaido University, Hokkaido, Japan; IMAB, Institute of Microbiology and Agropecurious Industry, Castelar, Argentina; FDA, Food and Drug Administration, Washington, D.C.; CCM, Czech Collection of Microorganisms, Masaryk University, Brno, Czech Republic; NCTC, National Collection of Type Cultures, Central Public Health Laboratory Service, London, United Kingdom; BUCSAV, Biologicky Ustav, Czeskslovenska Akademie Ved, Prague, Czech Republic; NCIB, National Collection of Industrial and Marine Bacteria, Ltd., Aberdeen, Scotland, United Kingdom; NCA, National Canners Association, Washington, D.C.; NRRL, Agricultural Research Service Culture Collection, National Center for Agricultural Utilization Research, Peoria, Ill.; IFO, Institute for Fermentation, Osaka, Japan; DSMZ and DSM, Deutsche Sammlung von Mikroorganismen und Zellkulturen GmbH, Braunschweig, Germany; ATCC, American Type Culture Collection, Rockville, Md.; IAM, Institute of Applied Microbiology, Tokyo, Japan; JCM, Japan Collection of Microorganisms, Saitama, Japan; CIP, Collection des Bactéries de l'Institut Pasteur, Paris, France; NRIC, NODAI Research Institute, Culture Collection Center, Tokyo University of Agriculture, Tokyo, Japan.

rRNA gene sequence similarity values greater than $89.6 \%$ (data not shown) placed the six Bacillus species, P. lautus, and $P$. peoriae within the realm of the genus Paenibacillus. In a phylogenetic tree, the two strains belonging to each of the six Bacillus species and the two Paenibacillus species were members of a robust monophyletic cluster containing the Paenibacillus species (Fig. 1). An inspection of the tree revealed close relationships between $P$. peoriae and $P$. azotofixans, between $B$. glucanolyticus and $P$. lautus, between $B$. thiaminolyticus and the $P$. alvei-P. apiarius complex, between $B$. curdlanolyticus and $B$. kobensis, and between $B$. alginolyticus and $B$. chondroitinus.

Cellular fatty acid compositions. A total of 47 strains of aerobic, endospore-forming rods were analyzed to determine their cellular fatty acid compositions. All of the strains in the
Paenibacillus cluster contained anteiso- $\mathrm{C}_{15: 0}$ acid as a major cellular fatty acid, and the level of this fatty acid ranged from 36.9 to $81.0 \%$ (Table 2).

Identifying members of the Paenibacillus cluster by $16 \mathrm{~S}$ rRNA gene amplification. Primer PAEN515F designed for detection of the Paenibacillus cluster spanned positions 491 to 515 (Fig. 2). PCR amplifications with primers PAEN515F and $1377 \mathrm{R}$ produced a 0.8 -kb PCR fragment in preparations of the strains of all of the members of the Paenibacillus cluster tested (including the six Bacillus species), but not in preparations of strains belonging to other clusters (Fig. 3 [only data for the type strains are shown]). A 1.3-kb fragment was amplified in all of the strains tested with primers $27 \mathrm{FC}$ and $1377 \mathrm{R}$ (data not shown).

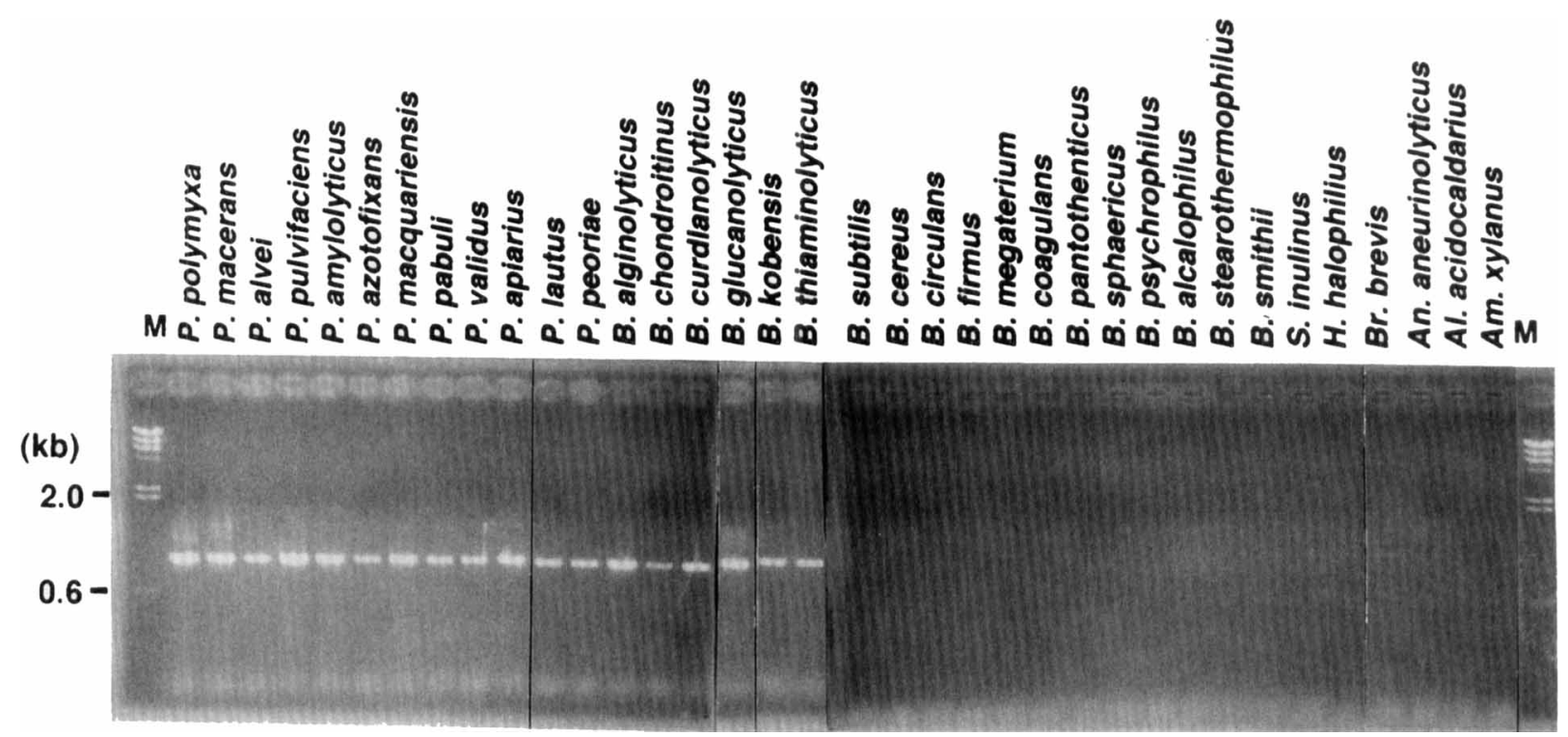

FIG. 3. Amplification of the 16S rRNA gene by PCR with detection primer PAEN515F and universal reverse primer 1377R. Results for only type strains are shown Lane M contained HindIII-digested $\lambda$ DNA as a molecular weight marker. Abbreviations: P., Paenibacillus; B., Bacillus; S., Sporolactobacillus; H., Halobacillus; Br., Brevibacillus; An., Aneurinibacillus; Al., Alicyclobacillus; Am., Amphibacillus. 
TABLE 2. Cellular fatty acid compositions of several species in the genera Bacillus and Paenibacillus and related organisms

\begin{tabular}{|c|c|c|c|c|c|c|c|c|c|c|c|c|c|c|c|}
\hline \multirow{4}{*}{ Strain } & \multicolumn{15}{|c|}{$\%$ of total cellular fatty acids } \\
\hline & \multicolumn{12}{|c|}{ Saturated acids } & \multicolumn{3}{|c|}{ Unsaturated acids } \\
\hline & \multicolumn{4}{|c|}{ Straight chain } & \multicolumn{5}{|c|}{ iso branched } & \multicolumn{3}{|c|}{ anteiso branched } & $C_{16: 1}$ & $C_{16: 1}$ & iso- $C_{17: 1}$ \\
\hline & $C_{13: 0}$ & $\mathrm{C}_{14: 0}$ & $C_{15: 0}$ & $\mathrm{C}_{16: 0}$ & $\mathrm{C}_{13: 0}$ & $\mathrm{C}_{14: 0}$ & $\mathrm{C}_{15: 0}$ & $C_{16: 0}$ & $\mathrm{C}_{17: 0}$ & $\mathrm{C}_{13: 0}$ & $\mathrm{C}_{15: 0}$ & $\mathrm{C}_{17 ; 0}$ & $\omega 9$ & $\omega 11$ & $\omega 10$ \\
\hline Bacillus alginolyticus strains & & & & & & & & & & & & & & & \\
\hline DSMZ $5050^{\mathrm{T}}$ & $\mathrm{ND}^{a}$ & 0.3 & 1.4 & 2.6 & ND & 1.2 & 4.7 & 10.4 & 1.7 & ND & 69.6 & 6.2 & ND & ND & ND \\
\hline NRRL NRS-1350 & ND & 0.2 & 1.1 & 3.1 & ND & 0.5 & 3.4 & 11.0 & 1.5 & ND & 73.0 & 5.4 & ND & ND & ND \\
\hline Bacillus chondroitinus strains & & & & & & & & & & & & & & & \\
\hline DSMZ $5051^{\mathrm{T}}$ & ND & 0.9 & 2.0 & 6.4 & ND & 1.7 & 2.4 & 10.4 & 0.7 & ND & 69.7 & 3.2 & ND & ND & ND \\
\hline NRRL B-14420 & ND & 0.5 & 0.9 & 5.0 & ND & 1.1 & 3.2 & 10.3 & 2.6 & ND & 67.4 & 5.3 & ND & ND & ND \\
\hline Bacillus curdlanolyticus strains & & & & & & & & & & & & & & & \\
\hline IFO $15724^{\mathrm{T}}$ & ND & 0.5 & 0.6 & 6.7 & ND & 1.6 & 1.7 & 24.4 & 0.8 & ND & 56.3 & 3.2 & ND & ND & ND \\
\hline IFO 15726 & ND & 0.4 & 0.9 & 5.0 & ND & 3.5 & 1.7 & 27.1 & 0.7 & ND & 56.7 & 3.0 & ND & ND & ND \\
\hline Bacillus glucanolyticus strains & & & & & & & & & & & & & & & \\
\hline DSMZ $5162^{\mathrm{T}}$ & ND & 0.8 & 0.7 & 11.2 & ND & 1.4 & 3.2 & 13.9 & 2.0 & ND & 56.5 & 8.2 & ND & 0.5 & 0.2 \\
\hline DSMZ 5188 & ND & 0.4 & 0.5 & 10.1 & ND & 0.7 & 1.0 & 12.1 & 1.6 & ND & 60.1 & 12.8 & ND & ND & 0.2 \\
\hline Bacillus kobensis strains & & & & & & & & & & & & & & & \\
\hline IFO $15729^{\mathrm{T}}$ & ND & 1.0 & 2.8 & 12.2 & ND & 0.5 & 2.0 & 8.8 & 0.5 & ND & 65.7 & 3.2 & ND & ND & ND \\
\hline IFO 15730 & ND & 0.7 & 2.7 & 8.3 & ND & 1.1 & 1.7 & 16.2 & 0.7 & ND & 61.1 & 4.1 & ND & ND & ND \\
\hline Bacillus thiaminolyticus strains & & & & & & & & & & & & & & & \\
\hline JCM $8630^{\mathrm{T}}$ & ND & 0.9 & 0.6 & 10.5 & ND & 0.6 & 10.5 & 5.8 & 6.5 & ND & 42.4 & 11.6 & ND & 5.2 & 1.7 \\
\hline JCM 7540 & ND & 0.5 & 0.5 & 8.5 & ND & 0.6 & 10.7 & 6.3 & 7.1 & ND & 45.3 & 16.4 & ND & 1.3 & 1.0 \\
\hline Paenibacillus alvei strains & & & & & & & & & & & & & & & \\
\hline IFO $3343^{\mathrm{T}}$ & ND & 2.3 & 1.6 & 15.3 & ND & 0.3 & 12.3 & 2.3 & 3.3 & ND & 53.9 & 3.3 & ND & 1.3 & 0.5 \\
\hline NRRL NRS-811 & ND & 1.8 & 2.0 & 12.2 & ND & 0.2 & 9.2 & 2.5 & 2.0 & ND & 60.3 & 4.0 & ND & 0.8 & 0.2 \\
\hline Paenibacillus amylolyticus strains & & & & & & & & & & & & & & & \\
\hline NRRL B-377 ${ }^{\mathrm{T}}$ & ND & 0.6 & 0.5 & 20.1 & ND & 0.2 & 2.9 & 4.1 & 5.8 & ND & 36.9 & 11.5 & ND & 0.5 & 1.0 \\
\hline NRRL B-142 & ND & 0.3 & 0.6 & 5.3 & ND & 0.8 & 4.0 & 12.2 & 3.2 & ND & 57.8 & 14.3 & ND & ND & ND \\
\hline Paenibacillus apiarius strains & & & & & & & & & & & & & & & \\
\hline NRRL NRS- $1438^{\mathrm{T}}$ & ND & 0.6 & 2.2 & 4.8 & ND & 0.2 & 8.3 & 4.2 & 5.5 & ND & 60.5 & 16.3 & ND & 0.8 & 0.2 \\
\hline NRRL NRS-1578 & ND & 0.5 & 1.4 & 4.6 & $\mathrm{ND}$ & 0.4 & 6.1 & 5.3 & 6.0 & ND & 59.3 & 16.3 & ND & 0.2 & 0.3 \\
\hline Paenibacillus azotofixans strains & & & & & & & & & & & & & & & \\
\hline NRRL B-14372 & ND & 1.5 & 0.3 & 17.6 & ND & 0.7 & 1.7 & 6.7 & 1.1 & ND & 62.2 & 5.0 & ND & 0.2 & ND \\
\hline NRRL B-14359 & ND & 0.9 & 0.2 & 15.2 & ND & 0.8 & 2.0 & 5.1 & 0.8 & ND & 60.8 & 3.5 & ND & 0.3 & ND \\
\hline $\begin{array}{c}\text { Paenibacillus larvae subsp. } \\
\text { pulvifaciens } \text { strains }\end{array}$ & & & & & & & & & & & & & & & \\
\hline IFO $15408^{\mathrm{T}}$ & ND & 0.6 & 1.3 & 6.4 & ND & 0.2 & 9.5 & 5.3 & 4.5 & ND & 48.8 & 20.7 & ND & 0.8 & ND \\
\hline NRRL B-14152 & ND & 0.2 & 1.6 & 4.7 & ND & 0.2 & 8.0 & 1.3 & 7.2 & ND & 40.8 & 30.2 & ND & 1.0 & ND \\
\hline Paenibacillus lautus strains & & & & & & & & & & & & & & & \\
\hline NRRL NRS-666 ${ }^{\mathrm{T}}$ & ND & 1.1 & 0.3 & 15.6 & ND & 0.8 & 1.5 & 7.4 & 1.2 & ND & 57.3 & 9.7 & ND & 2.0 & 0.2 \\
\hline NRRL B-379 & ND & 0.5 & 0.3 & 18.3 & ND & 0.4 & 2.6 & 3.6 & 6.0 & ND & 34.8 & 10.2 & ND & 0.3 & 0.4 \\
\hline Paenibacillus macerans strains & & & & & & & & & & & & & & & \\
\hline JCM $2500^{\mathrm{T}}$ & ND & 3.7 & 0.5 & 17.9 & ND & 7.9 & 2.6 & 16.4 & 0.6 & ND & 36.1 & 12.2 & ND & 0.1 & ND \\
\hline IAM 1243 & ND & 1.9 & 3.1 & 17.4 & ND & 1.8 & 2.6 & 17.1 & 4.1 & ND & 34.5 & 16.1 & ND & 0.1 & ND \\
\hline Paenibacillus macquariensis strains & & & & & & & & & & & & & & & \\
\hline CIP $103269^{\mathrm{T}}$ & ND & 0.7 & 1.2 & 2.9 & ND & 0.8 & 5.2 & 2.6 & 0.4 & ND & 81.0 & 0.7 & ND & 0.6 & 0.2 \\
\hline NRRL NRS-1534 & ND & 0.4 & 1.5 & 1.5 & ND & 1.0 & 4.1 & 4.1 & 0.2 & ND & 78.2 & 1.0 & ND & 0.3 & 0.3 \\
\hline Paenibacillus pabuli strains & & & & & & & & & & & & & & & \\
\hline NRRL NRS- $924^{T}$ & ND & 0.7 & 0.1 & 10.1 & ND & 0.7 & 2.2 & 4.8 & 1.3 & ND & 73.7 & 4.1 & ND & ND & ND \\
\hline NRRL BD-537 & ND & 0.8 & 0.4 & 11.0 & ND & 3.9 & 1.7 & 7.7 & 0.8 & ND & 69.8 & 3.0 & ND & ND & ND \\
\hline Paenibacillus peoriae strains & & & & & & & & & & & & & & & \\
\hline IFO $15541^{\mathrm{T}}$ & ND & 0.7 & 0.5 & 10.6 & ND & 1.4 & 7.9 & 6.7 & 5.0 & ND & 54.7 & 10.5 & ND & 0.5 & 0.3 \\
\hline NRRL B-14476 & ND & 0.8 & 0.3 & 9.3 & ND & 0.9 & 3.5 & 6.7 & 6.0 & ND & 60.9 & 10.9 & ND & ND & ND \\
\hline Paenibacillus polymyxa strains & & & & & & & & & & & & & & & \\
\hline $\mathrm{JCM} 2507^{\mathrm{T}}$ & ND & 0.4 & 0.3 & 9.3 & ND & 0.5 & 1.0 & 5.6 & 1.6 & ND & 62.9 & 16.9 & ND & ND & ND \\
\hline NRRL BD-55 & ND & 0.5 & 0.3 & 11.0 & ND & 0.4 & 0.2 & 4.5 & 1.1 & ND & 51.0 & 28.8 & ND & ND & ND \\
\hline Paenibacillus validus strains & & & & & & & & & & & & & & & \\
\hline DSMZ $3037^{\mathrm{T}}$ & ND & 0.7 & 1.4 & 10.8 & ND & 1.4 & 4.1 & 11.7 & 3.4 & ND & 57.3 & 7.5 & ND & 0.4 & 0.1 \\
\hline CIP 103498 & ND & 0.9 & 0.7 & 10.2 & ND & 1.6 & 6.8 & 9.1 & 3.4 & ND & 58.7 & 4.9 & ND & 0.5 & 0.2 \\
\hline Bacillus subtilis JCM $1465^{\mathrm{T}}$ & ND & 0.3 & 2.3 & 1.3 & ND & 0.4 & 28.4 & 2.0 & 6.1 & ND & 44.5 & 6.8 & 3.3 & ND & 0.5 \\
\hline Bacillus cereus JCM $2152^{\mathrm{T}}$ & 0.8 & 3.1 & 4.9 & 2.4 & 7.8 & 2.4 & 48.7 & 2.7 & 6.2 & 0.6 & 3.8 & 0.7 & 1.1 & 4.4 & 2.8 \\
\hline Bacillus megaterium JCM $2506^{\mathrm{T}}$ & ND & 1.7 & 1.8 & 1.6 & ND & 8.2 & 32.6 & 1.1 & 0.4 & ND & 45.2 & 1.1 & ND & ND & ND \\
\hline Bacillus circulans IAM $12462^{\mathrm{T}}$ & ND & 4.3 & 2.2 & 3.7 & ND & 3.9 & 10.0 & 3.5 & ND & ND & $57: 3$ & 3.4 & ND & ND & ND \\
\hline Bacillus badius ATCC $14574^{\mathrm{T}}$ & ND & 2.1 & 9.6 & 2.4 & ND & 1.6 & 60.0 & 3.2 & 1.7 & ND & 7.6 & 1.8 & ND & ND & ND \\
\hline Bacillus sphaericus JCM $2502^{\mathrm{T}}$ & ND & 1.4 & 6.8 & 1.3 & ND & 6.0 & 53.4 & 6.9 & 2.1 & ND & 8.8 & 0.9 & ND & ND & ND \\
\hline Bacillus stearothermophilus JCM $2501^{\mathrm{T}}$ & ND & ND & ND & 3.4 & ND & ND & 27.5 & 5.6 & 17.2 & ND & 7.8 & 38.6 & ND & ND & ND \\
\hline Bacillus coagulans JCM $2257^{\mathrm{T}}$ & ND & 0.5 & 5.3 & 0.7 & ND & ND & 2.2 & 0.4 & ND & ND & 65.8 & 22.6 & ND & ND & ND \\
\hline Sporolactobacillus inulinus NRIC $1133^{\mathrm{T}}$ & ND & ND & 5.1 & 0.8 & ND & ND & 1.7 & 0.8 & ND & ND & 52.9 & 35.2 & ND & ND & ND \\
\hline Brevibacillus brevis JCM $2503^{\mathrm{T}}$ & ND & 0.4 & 0.7 & 2.7 & ND & 0.6 & 17.6 & 3.5 & 5.3 & ND & 54.4 & 9.6 & ND & ND & ND \\
\hline $\begin{array}{l}\text { Aneurinibacillus aneurinolyticus } \\
\text { ATCC } 12856^{\mathrm{T}}\end{array}$ & ND & 0.8 & 1.0 & 5.9 & ND & 0.5 & 44.7 & 13.9 & 6.3 & ND & 4.4 & 1.1 & ND & 4.1 & 7.8 \\
\hline
\end{tabular}

${ }^{a} \mathrm{ND}$, not detected. 


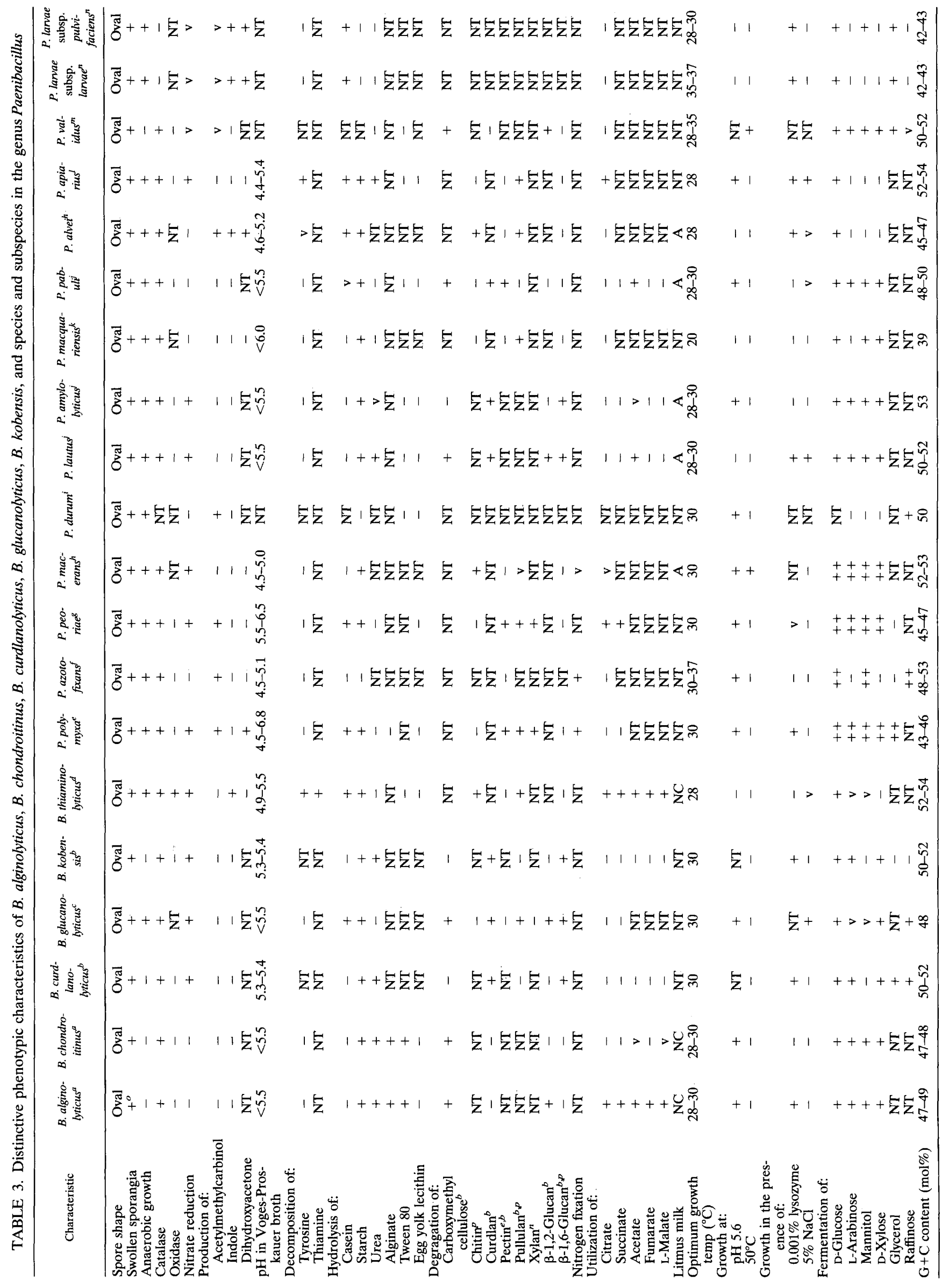




\section{DISCUSSION}

A phylogenetic analysis based on 16S rRNA gene sequences placed $B$. alginolyticus, $B$. chondroitinus, $B$. curdlanolyticus, $B$. glucanolyticus, $B$. kobensis, $B$. thiaminolyticus, $P$. lautus, and $P$. peoriae in a robust (bootstrap value, 1,000 ) cluster consisting of the Paenibacillus species. Sequence comparisons performed with members of this cluster revealed intracluster similarity values greater than $89.6 \%$. The results of the phylogenetic analysis of $B$. thiaminolyticus, $P$. lautus, and $P$. peoriae correlated well with the results of polyphasic taxonomy reported by Heyndrickx et al. (13). Ash et al. (2) reported that $P$. lautus (formerly B. lautus) was a member of rRNA group 1 based on the 16S rRNA sequence of the type strain. The different position of this species may have been due to an erroneous gene sequence used in the study of Ash et al. (2).

Table 3 shows phenotypic characteristics of the members of the Paenibacillus cluster. Interestingly, species that formed groups in the Paenibacillus cluster exhibited similar polysaccharidase or physiological activities or originated from com mon habitats. For example, $P$. peoriae, $P$. azotofixans, and $P$. polymyxa are members of a group (Fig. 1) that hydrolyze pectin and xylan and fix nitrogen. The $B$. glucanolyticus- $P$. lautus, $B$. curdlanolyticus- $B$. kobensis, and $B$. alginolyticus- $B$. chondroitinus pairs are closely related organisms that share the ability to hydrolyze various $\beta$-glucans, curdlan, and alginate, respectively. $B$. thiaminolyticus, $P$. alvei, and $P$. apiarius appear to be members of a group (Fig. 1) that consists of species which are frequently isolated from honeybee environs.

The general similarity of the distribution of cellular fatty acids in the organisms also indicates the cohesiveness of the Paenibacillus cluster (Fig. 3). Furthermore, like many previously described Paenibacillus species, the new species exhibit diverse polysaccharidase activities $(1,14,19,25)$.

Detection primer PAEN515F was designed on the basis of a signature DNA sequence found in the 16S rRNA gene of Paenibacillus species. Ash et al. (2) showed that slot blot hybridization is useful for identification of the genus Paenibacillus when an amplified 16S rRNA gene from chromosomal DNA and a specific probe are used. Shida et al. (31) described a rapid, simple, and efficient method for identifying the genera Brevibacillus and Aneurinibacillus by PCR amplification of $16 \mathrm{~S}$ rRNA gene fragments with genus-specific detection primers. The genus Paenibacillus was successfully differentiated from the other taxa belonging to the Bacillaceae by using PAEN515F as the detection primer. Concomitantly, the six Bacillus species placed in the Paenibacillus cluster were identified as members of the genus Paenibacillus. At this time the phenotypic characteristics available for differentiating and identifying the genera belonging to the Bacillaceae are inadequate (Table 4). The use of probes and specific detection primers should be very useful for identifying and differentiating members of the Bacillaceae.

Based on the observations described above, we propose that $B$. alginolyticus, $B$. chondroitinus, $B$. curdlanolyticus, $B$. glucanolyticus, $B$. kobensis, and $B$. thiaminolyticus should be transferred to the genus Paenibacillus as Paenibacillus alginolyticus, Paenibacillus chondroitinus, Paenibacillus curdlanolyticus, Paenibacillus glucanolyticus, Paenibacillus kobensis, and Paenibacillus thiaminolyticus, respectively.

Description of Paenibacillus alginolyticus (Nakamura 1987) comb. nov. The description of Paenibacillus alginolyticus comb. nov. is identical to the description of $B$. alginolyticus given by Nakamura (19). The major cellular fatty acids are anteiso- $C_{15: 0}$ and iso- $\mathrm{C}_{16: 0}$ acids (this study). Type strain DSMZ 5050 $(=$ NRRL NRS-1347 $=$ ATCC $51185=$ CIP $103122=$ IFO 


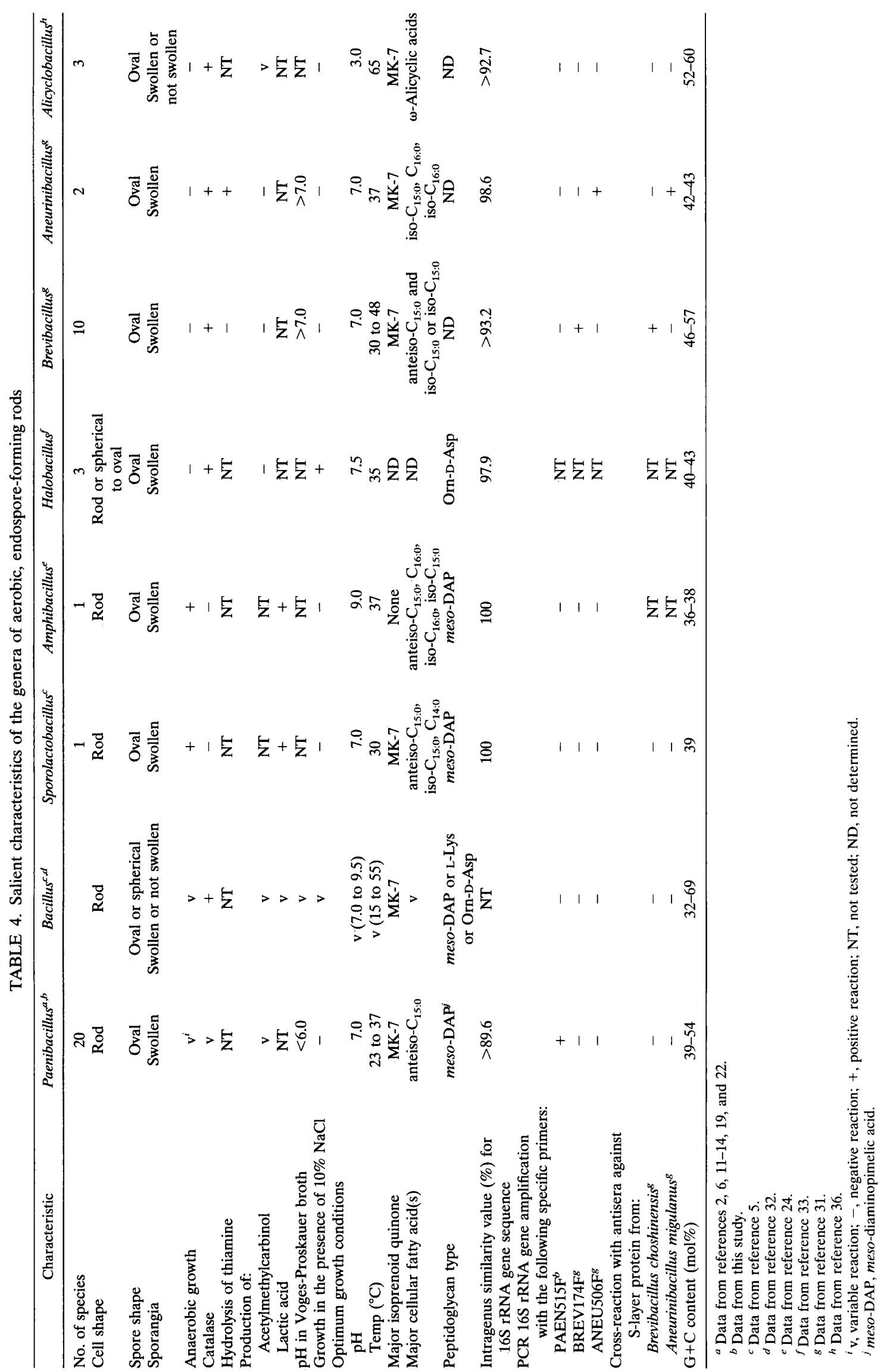


$15375=$ JCM $9068=$ NCIMB 12517) has been deposited in the Culture Collection of the Research Laboratory of Higeta Shoyu Co., Ltd. as strain HSCC 175.

Description of Paenibacillus chondroitinus (Nakamura 1987) comb. nov. The description of Paenibacillus chondroitinus comb. nov. is identical to the description of $B$. chondroitinus given by Nakamura (19). The major cellular fatty acids are anteiso- $\mathrm{C}_{15: 0}$ and iso- $\mathrm{C}_{16: 0}$ acids (this study). Type strain DSMZ 5051 (= NRRL NRS-1351 = ATCC $51184=$ IFO $15376=$ JCM 9072) has been deposited in the Culture Collection of the Research Laboratory of Higeta Shoyu Co., Ltd. as strain HSCC 176.

Description of Paenibacillus curdlanolyticus (Kanzawa et al. 1995) comb. nov. The description of Paenibacillus curdlanolyticus comb. nov. is identical to the description of $B$. curlanolyticus given by Kanzawa et al. (14). Type strain IFO 15724 $(=$ ATCC $51898=$ CIP 104575) has been deposited in the Culture Collection of the Research Laboratory of Higeta Shoyu Co., Ltd. as strain HSCC 491.

Description of Paenibacillus glucanolyticus (Alexander and Priest 1989) comb. nov. The description of Paenibacillus glucanolyticus comb. nov. is identical to the description of $B$. glucanolyticus given by Alexander and Priest (1). The major cellular fatty acids are anteiso- $\mathrm{C}_{15: 0}$ and iso- $\mathrm{C}_{15: 0}$ acids (this study). Type strain DSMZ 5162 (= IFO 15330 = NCIMB 12809) has been deposited in the Culture Collection of the Research Laboratory of Higeta Shoyu Co., Ltd. as strain HSCC 171.

Description of Paenibacillus kobensis (Kanzawa et al. 1995) comb. nov. The description of Paenibacillus kobensis comb. nov. is identical to the description of $B$. kobensis given by Kanzawa et al. (14). Type strain IFO 15729 (= ATCC $51900=$ CIP 104576) has been deposited in the Culture Collection of the Research Laboratory of Higeta Shoyu Co., Ltd. as strain HSCC 488.

Description of Paenibacillus thiaminolyticus (Nakamura 1990) comb. nov. The description of Paenibacillus thiaminolyticus comb. nov. is identical to the description of $B$. thiaminolyticus given by Nakamura (21). The major cellular fatty acids are anteiso- $\mathrm{C}_{15: 0}$, anteiso- $\mathrm{C}_{17: 0}, \mathrm{C}_{16: 0}$, and iso- $\mathrm{C}_{15: 0}$ acids (this study). Type strain JCM 8360 (= AHU $1393=$ NRRL B-4156 = IFO 15656) has been deposited in the Culture Collection of the Research Laboratory of Higeta Shoyu Co., Ltd. as strain HSCC 148.

Emendation of the genus Paenibacillus. The observations made in this study revealed that the genus Paenibacillus consists of 19 species and one subspecies. Some characteristics in the original description of this genus as proposed by Ash et al. (2) were not found in several Paenibacillus species. Therefore, the description of this genus should be emended, as described below.

Emended description of the genus Paenibacillus Ash, Priest, and Collins 1993. Cells are rod shaped. Gram positive, gram negative, or gram variable. Motile by means of peritrichous flagella. Ellipsoidal spores are formed in swollen sporangia. No soluble pigment is produced on nutrient agar.

Facultatively anaerobic or strictly aerobic.

Almost all of the species are positive for catalase. Paenibacillus larvae subsp. larvae and Paenibacillus larvae subsp. pulvifaciens are negative for catalase. Oxidase activity is variable.

The Voges-Proskauer reaction (production of acetylmethylcarbinol) is variable, and the $\mathrm{pH}$ in Voges-Proskauer broth is less than 6.0 .

Hydrogen sulfide is not produced. Indole is produced by some species.

Nitrate reduction to nitrite is variable.
Hydrolysis of casein, hydrolysis of starch, and hydrolysis of urea are variable.

Decomposition of tyrosine is variable.

Growth at $\mathrm{pH} 5.6$ and growth at $50^{\circ} \mathrm{C}$ are variable. Optimum growth occurs at $\mathrm{pH}$ 7.0. The optimum growth temperature of 19 species (all species except $P$. macquariensis) is 28 to $30^{\circ} \mathrm{C}$ The optimum growth temperature of $P$. macquariensis is 20 to $23^{\circ} \mathrm{C}$. Growth is inhibited by $10 \% \mathrm{NaCl}$. Some species do not grow in medium containing $0.001 \%$ lysozyme.

Acid is produced from various sugars. $P$. polymyxa, $P$. peoriae, $P$. azotofixans, and $P$. macerans produce gas from various sugars.

Some species decompose polysaccharides.

The major cellular fatty acid is anteiso- $\mathrm{C}_{15: 0}$ acid.

The $\mathrm{G}+\mathrm{C}$ contents range from 45 to $54 \mathrm{~mol} \%$.

The levels of 16S rRNA gene sequence similarity are more than $89.6 \%$ for the members of this genus. A 16S rRNA gene fragment is amplified by PCR with primers PAEN515F and 1377R.

The type species is $P$. polymyxa.

\section{ACKNOWLEDGMENTS}

We thank K. Sano, H. Shimada, and R. Wakamatsu for technical assistance.

\section{REFERENCES}

1. Alexander, B., and F. G. Priest. 1989. Bacillus glucanolyticus, a new species that degrades a variety of $\beta$-glucans. Int. J. Syst. Bacteriol. 39:112-115.

2. Ash, C., F. G. Priest, and M. D. Collins. 1993. Molecular identification of rRNA group 3 bacilli (Ash, Farrow, Wallbanks, and Collins) using a PCR probe test. Proposal for the creation of a new genus Paenibacillus. Antonie van Leeuwenhoek 64:253-260.

3. Ash, C., J. A. E. Farrow, S. Wallbanks, and M. D. Collins. 1991. Phylogenetic heterogeneity of the genus Bacillus revealed by comparative analysis of small-subunit-ribosomal RNA sequence. Lett. Appl. Microbiol. 13:202-206.

4. Cato, E. P., W. L. George, and S. M. Finegold. 1986. Genus Clostridium Prazmowski 1880, p. 1141-1200. In P. H. A. Sneath, N. S. Mair, M. E. Sharpe, and J. G. Holt (ed.), Bergey's manual of systematic bacteriology, vol. 2. The Williams and Wilkins Co., Baltimore, Md.

5. Claus, D., and R. C. W. Berkeley. 1986. Genus Bacillus Cohn 1872, p. 1105-1140. In P. H. A. Sneath, N. S. Mair, M. E. Sharpe, and J. G. Holt (ed.), Bergey's manual of systematic bacteriology, vol. 2. The Williams and Wilkins Co., Baltimore, Md.

6. Collins, M. D., P. A. Lawson, A. Willems, J. J. Cordoba, J. FernandezGarayzabal, P. Garcia, J. Cai, H. Hippe, and J. A. E. Farrow. 1994. The phylogeny of the genus Clostridium: proposal of five new genera and eleven new species combinations. Int. J. Syst. Bacteriol. 44:812-826.

7. Farrow, J. A. E., S. Wallbanks, and M. D. Collins. 1992. Phylogenetic analysis of the genera Planococcus, Marinococcus, and Sporosarcina and thei relationship to members of the genus Bacillus. FEMS Microbiol. Lett. 93: 167-172.

8. Felsenstein, J. 1985. Confidence limits on phylogenies: an approach using the bootstrap. Evolution 39:783-791.

9. Fox, G. E., J. Wisotzkey, and P. Jurtshuk, Jr. 1992. How close is close: 16S rRNA sequence identity may not be sufficient to guarantee species identity. Int. J. Syst. Bacteriol. 42:166-170.

10. Gordon, R. E., W. C. Hynes, and C. H. N. Pang. 1973. The genus Bacillus. Agricultural Handbook no. 427. U.S. Department of Agriculture, Washington, D.C.

11. Heyndrickx, M., K. Vandemeulebroecke, B. Hoste, P. Janssen, K. Kersters, P. De Vos, N. A. Logan, N. Ali, and R. C. W. Berkeley. 1996. Reclassification of Paenibacillus (formerly Bacillus) pulvifaciens (Nakamura 1984) Ash et al. 1994, a later subjective synonym of Paenibacillus (formerly Bacillus) larvae (White 1966) Ash et al. 1994, as a subspecies of $P$ larvae, with emended description of $P$. larvae as $P$. larvae subsp. larvae and $P$. larvae subsp. pulvifaciens. Int. J. Syst. Bacteriol. 46:270-279.

12. Heyndrickx, M., K. Vandemeulebroecke, P. Scheldeman, B. Hoste, K. Kersters, P. De Vos, N. A. Logan, A. M. Aziz, N. Ali, and R. C. W. Berkeley. 1995 Paenibacillus (formerly Bacillus) gordonae (Pichinoty et al. 1986) Ash et al. 1994 is a later subjective synonym of Paenibacillus (formerly Bacillus) validus (Nakamura 1984) Ash et al. 1994: emended description of $P$. validus. Int. J. Syst. Bacteriol. 45:661-668.

13. Heyndrickx, M., K. Vandemeulebroecke, P. Scheldeman, K. Kersters, P. De Vos, N. A. Logan, A. M. Aziz, N. Ali, and R. C. W. Berkeley. 1996. A polyphasic reassessment of the genus Paenibacillus, reclassification of Bacil- 
lus lautus (Nakamura 1984) as Paenibacillus lautus comb, nov, and Bacillus peoriae (Montefusco et al. 1993) as Paenibacillus peoriae comb. nov., and emended description of $P$. lautus and $P$. peoriae. Int. J. Syst. Bacteriol. 46:988-1003.

14. Kanzawa, Y., A. Harada, M. Takeuchi, A. Yokota, and T. Harada. 1995 Bacillus curdlanolyticus sp. nov. and Bacillus kobensis sp. nov., which hydrolyze resistant curdlan. Int. J. Syst. Bacteriol. 45:515-521.

15. Kimura, M. 1980. A simple method for estimating evolutionary rates of base substitutions through comparative studies of nucleotide sequences. J. Mol Evol. 16:111-120.

16. Komagata, K., and K. Suzuki. 1987. Lipid and cell-wall analysis in bacterial systematics. Methods Microbiol. 19:161-207.

17. Montefusco, A., L. K. Nakamura, and D. P. Labeda. 1993. Bacillus peoriae sp. nov. Int. J. Syst. Bacteriol. 43:388-390.

18. Nakamura, L. K. 1984. Bacillus amylolyticus sp. nov., nom. rev., Bacillus lautus sp. nov., nom. rev., Bacillus pabuli sp. nov., nom. rev., and Bacillus validus sp. nov., nom. rev. Int. J. Syst. Bacteriol. 34:224-226.

19. Nakamura, L. K. 1987. Bacillus alginolyticus $\mathrm{sp}$. nov. and Bacillus chondroitinus sp. nov., two alginate-degrading species. Int. J. Syst. Bacteriol. 37:284-286.

20. Nakamura, L. K. 1987. Bacillus polymyxa (Prazmowski) Mace 1889 deoxyribonucleic acid relatedness and base composition. Int. J. Syst. Bacteriol. 37:391-397.

21. Nakamura, L. K. 1990. Bacillus thiaminolyticus sp. nov., nom. rev. Int. J. Syst. Bacteriol. 40:242-246.

22. Nakamura, L. K. 1996. Paenibacillus apiarius sp. nov., nom. rev. Int. J. Syst. Bacteriol. 46:688-693.

23. Nielsen, P., F. A. Rainey, F. A. Outtrup, F. G. Priest, and D. Fritze. 1994 Comparative 16S rDNA sequence analysis of some alkaliphilic bacilli and the establishment of a sixth rRNA group within the genus Bacillus. FEMS Microbiol. Lett. 117:61-66.

24. Niimura, Y., E. Koh, F. Yanagida, K. Suzuki, K. Komagata, and M. Kozaki. 1990. Amphibacillus xylanus gen. nov., sp. nov., a facultatively anaerobic sporeforming xylan-digesting bacterium which lacks cytochrome, quinone, and catalase. Int. J. Syst. Bacteriol. 40:297-301.

25. Priest, F. G., M. Goodfellow, and C. Todd. 1988. A numerical classification of the genus Bacillus. J. Gen. Microbiol. 134:1847-1882.

26. Rainey, F. A., D. Fritze, and E. Stackebrandt. 1994. The phylogenetic diver- sity of thermophilic members of the genus Bacillus as revealed by 16S rDNA analysis. FEMS Microbiol. Lett. 115:205-212.

27. Russler, D., W. Ludwig, K. H. Schleifer, C. Lin, T. J. McGill, J. D. Wisotzkey, P. Jurtshuk, Jr., and G. E. Fox. 1991. Phylogenetic diversity in the genus Bacillus as seen by $16 \mathrm{~S}$ rRNA sequencing studies. Syst. Appl. Microbiol. 14:266-269.

28. Saito, N., and M. Nei. 1987. A neighbor-joining method: a new method for reconstructing phylogenetic trees. Mol. Biol. Evol. 4:406-425.

29. Sanger, F., S. Nicklen, and A. R. Coulson. 1977. DNA sequencing with chain-terminating inhibitors. Proc. Natl. Acad. Sci. USA 74:5463-5467.

30. Seldin, L., J. D. Van Elsas, and E. G. L. Penido. 1984. Bacillus azotofixans sp. nov., a nitrogen-fixing species from Brazilian soil and grass roots. Int. J. Syst. Bacteriol. 34:451-456

31. Shida, O., H. Takagi, K. Kadowaki, and K. Komagata. 1996. Proposal for two new genera, Brevibacillus gen. nov. and Aneurinibacillus gen. nov. Int. J. Syst. Bacteriol. 46:939-946.

32. Spanka, R., and D. Fritze. 1993. Bacillus cohnii sp. nov., a new, obligately alkaliphilic, oval-spore-forming Bacillus species with ornithine and aspartic acid instead of diaminopimelic acid in the cell wall. Int. J. Syst. Bacteriol. 43:150-156.

33. Spring, S., W. Ludwig, M. C. Marquez, A. Ventosa, and K.-H. Schleifer 1996. Halobacillus gen. nov., with description of Halobacillus litoralis $\mathrm{sp}$. nov. and Halobacillus truperi sp. nov., and transfer of Sporosarcina halophilia to Halobacillus halophilus comb. nov. Int. J. Syst. Bacteriol. 46:492496.

34. Suzuki, T., and K. Yamasato. 1994. Phylogeny of spore-forming lactic acid bacteria based on 16S rRNA gene sequences. FEMS Microbiol. Lett. 115: 13-18.

35. Thompson, J. D., D. G. Higgins, and T. J. Gibson. 1994. CLUSTAL W improving the sensitivity of progressive multiple sequence alignment through sequence weighting, position-specific gap penalties and weight matrix choice. Nucleic Acids Res. 22:4673-4680.

36. Wisotzkey, J. D., P. Jurtshuk, Jr., G. E. Fox, G. Deinhard, and K. Poralla 1992. Comparative sequence analysis on the 16S rRNA (rDNA) of Bacillus acidocaldarius, Bacillus acidoterrestris, and Bacillus cycloheptanicus and proposal for creation of a new genus, Alicyclobacillus gen. nov. Int. J. Syst. Bacteriol. 42:263-269. 